\title{
Diffusion-Controlled Recrystallization of Water Sorbed into Poly(meth)acrylates Revealed by Variable Temperature Mid-infrared Spectroscopy and Molecular Dynamics Simulation
}

\author{
${ }^{\dagger}$ Department of Applied Chemistry, Graduate School of Science and Engineering, \\ University of Toyama, Gofuku 3190, Toyama 930-8555, Japan \\ Institute for Polymer-Water Interfaces, 84 Fukujima, Yatsuo, Toyama 939-2376, Japan
}

Nobuhiro Yasoshima, ${ }^{\dagger}$ Mizuki Fukuoka, ${ }^{\dagger}$ Hiromi Kitano, ${ }^{\ddagger}$ Shigehiro Kagaya,${ }^{\dagger}$
Tatsuya Ishiyama, ${ }^{*, \dagger}$ and Makoto Gemmei-Ide, ${ }^{* \dagger}$

\begin{abstract}
* To whom correspondence should be addressed.
T. I.: e-mail: ishiyama@eng.u-toyama.ac.jp

M. G.-I: e-mail: ide@eng.u-toyama.ac.jp
\end{abstract}

\begin{abstract}
Recrystallization behaviors of water sorbed into four poly(meth)acrylates; poly(2-methoxyethyl acrylate) (PMEA), poly(tetrahydrofurfuryl acrylate) (PTHFA), poly(methyl acrylate) (PMA), and poly(methyl methacrylate) (PMMA), are investigated by variable-temperature mid-infrared (VT-MIR) spectroscopy and molecular dynamics (MD) simulation. VT-MIR spectra demonstrate that recrystallization temperatures of water sorbed into the polymers are positively correlated with their glass transition temperatures reported previously. The present MD simulation shows that a lower limit temperature of the diffusion for the sorbed water and the glass transition temperatures of the polymers also have a positive correlation, indicating that the recrystallization is controlled by diffusion mechanism rather than reorientation mechanism. Detailed molecular processes not only of recrystallization during rewarming but also of crystallization during cooling and hydrogen-bonding states of water in the polymers are systematically analyzed and discussed.
\end{abstract}

\section{Introduction}

Recrystallization of water, which is a crystallization during rewarming at a temperature sufficiently lower than the melting point of water $T_{\mathrm{m}}(=273.15 \mathrm{~K})$, is typically observed in various aqueous systems, and is of fundamental importance for understanding of water-material interaction. ${ }^{1}$ Recrystallization of pure water is known to take place at the temperature of $\sim 150 \mathrm{~K}^{2,3}$ due to devitrification of amorphous ice (also called as glassy water or vitrified water). The recrystallization phenomenon in water-polymer binary systems has also been examined in a wide range of water concentration. In particular, a measurement of the onset temperature of crystallization during rewarming (recrystallization temperature, hereafter referred to as $T_{\text {rc }}$ ), for a water-containing polymer at low water content provides a key information about the interaction of water contacting with the polymer. Molecular mechanism of recrystallization remains to be resolved in detail, though it is known that $T_{\mathrm{rc}}$ depends on chemical structures of polymer, water content, and cooling rate before rewarming. In this study, we examine behavior of water sorbed into four solid polymers such as poly(2-methoxyethyl acrylate) (PMEA), poly(tetrahydrofurfuryl acrylate) (PTHFA), poly(methyl acrylate) (PMA), and poly(methyl methacrylate) (PMMA), by variable-temperature mid-infrared (VT-MIR) spectroscopy and molecular dynamics (MD) simulation, and discuss recrystallization mechanism in detail.

(Re)Crystallization of water in the polymer-water binary systems has been widely observed by differential scanning calorimetry (DSC). ${ }^{4-11}$ DSC probes a heat flux from a sample with temperature perturbation and has been most frequently used because of its relatively simple operation and easy interpretation of given data. (Re)Crystallization of water is detected as an exothermic peak in a DSC curve and in addition a glass transition temperature, $T_{\mathrm{g}}^{\mathrm{DSC}}$, is also detected as a baseline shift. The previous DSC study reported that water-containing PMEA ${ }^{10}$ and PTHFA ${ }^{11}$ systems, which are non-water-soluble polymers, show a clear exothermic peak at $223 \mathrm{~K}$ and $248 \mathrm{~K}$ during rewarming after cooling to $173 \mathrm{~K}$, respectively, indicating the recrystallization in each polymer matrix. There are two possible scenarios to account for this observation: one is due to transition (reorientation) of condensed water formed in a polymer from a glass to a crystal (reorientation mechanism ${ }^{10,11}$ ), and the other is due to transition (deposition) from a monomolecular water trapped on a polymer chain to a crystal after diffusion (diffusion mechanism ${ }^{12-14}$ ). These two mechanisms are indistinguishable in the DSC experiment because only a net heat flux accompanying the state transitions of water is observed, and detailed molecular processes are missing in the observed exothermic peak.

Variable temperature mid-infrared (VT-MIR) spectroscopy is a powerful measure to know molecular environment confined in a polymer matrix, and has been developed by our group to demonstrate recrystallization of water sorbed into some non-water-soluble polymers such as PMEA, ${ }^{12}$ PMA, ${ }^{14}$ poly(ethoxyethyl acrylate) (PEEA), ${ }^{14}$ poly(ethoxyethyl 
methacrylate) (PEEMA), ${ }^{14}$ and polystyrene (PS). ${ }^{15}$ The previous VT-MIR measurements suggested that the recrystallized water in the above-mentioned six polymers was formed not due to the reorientation mechanism but due to the diffusion mechanism because an intense vibrational spectroscopic response at about $3250 \mathrm{~cm}^{-1}$, a signature of the formation of ice, was observed with decreasing the monomolecular water during rewarming process. However, the vibrational spectroscopy is an indirect measure of molecular motion, and further research is needed to understand the time-dependent process hidden behind the recrystallization in molecular details.

Molecular dynamics (MD) computer simulation technique is a promising way to discuss static and dynamic structures in molecular resolution and time-dependent processes such as diffusion. The recent MD studies containing aqueous polymer systems have mainly shed light on the interaction between water and polymer at a flat interface ${ }^{16}$. The results deduced from MD simulation, however, are sometimes dependent on simulation conditions such as force field employed, system size, initial and boundary conditions. Furthermore, a time scale accessible by MD simulation is usually limited within nano- or micro-second order for a polymer systems. Thus a close collaboration with experimental study is a key step to reach a definite answer to the above-mentioned question about which mechanism is valid in the recrystallization process.

In this paper, we systematically discuss molecular mechanism of recrystallization process in the four polymers by performing a collaborative study of VT-MIR spectroscopy and MD simulation. In the present VT-MIR experiment, O-H stretching vibration of water sorbed into the polymer matrices are measured as a function of temperature, and characteristic features of the MIR response during recrystallization are clarified. A correlation relation of $T_{\mathrm{rc}}$ and $T_{\mathrm{g}}^{\mathrm{DSC}}$ is found in the experiment. On the other hand, in the present $\mathrm{MD}$ simulation, glass transition temperature of the polymers, $T_{\mathrm{g}}^{\mathrm{MD}}$, and transition temperature of water diffusion in the polymers, $T_{\mathrm{D}}^{\mathrm{MD}}$, are examined by calculating specific volume of MD system and diffusion coefficient of the sorbed water as a function of temperature. Another correlation relation of $T_{\mathrm{D}}^{\mathrm{MD}}$ and $T_{\mathrm{g}}^{\mathrm{MD}}$ is found in the simulation. From these analyses, we elucidate the recrystallization process for the present water-containing polymers is controlled by the diffusion mechanism rather than the reorientation mechanism. We focus not only on recrystallization process during rewarming but also on crystallization process during cooling to discuss detailed transition processes of water sorbed in the polymers. Hydrogen bonding (H-bonding) states of water interacting with functional groups in the polymers are analyzed as a function of temperature to unveil time-dependent molecular processes during the recrystallization by MD simulation.

\section{Experiments and simulations}

2.1. Materials. PMEA, PTHFA, and PMA were prepared by a conventional free radical polymerization of corresponding monomer. Their weight-averaged molecular weights $\left(M_{\mathrm{w}}\right)$ and polydispersity indices $\left(M_{\mathrm{w}} / M_{\mathrm{n}}\right)$ were determined to be $8.6 \times 10^{4}$ and $1.7,5.8 \times 10^{4}$ and 2.1 , and $7.7 \times 10^{4}$ and 1.9 , respectively, by gel permeation chromatography. PMMA with $M_{\mathrm{w}}$ of $9.0 \times 10^{5}$ and $M_{\mathrm{w}} / M_{\mathrm{n}}$ of 1.4 was purchased from Wako Pure Chemicals, Osaka, Japan. The chemical structures of the four polymers are illustrated in Figure 1. The quality of water used in all experiments was Milli-Q grade (resistivity $>18 \mathrm{M} \Omega \cdot \mathrm{cm}$ ).

2.2. Mid-infrared Spectroscopy MIR spectra of the hydrated and dried polymers were recorded on a Spectrum One NTS (Perkin-Elmer) with a resolution of $4 \mathrm{~cm}^{-1}$ and a scanning time of $20 \mathrm{~s}$. Cooling rates were $0.5,1.0$, and $4.8 \mathrm{~K} \cdot \mathrm{min}{ }^{-1}$ and heating rate was $0.5 \mathrm{~K} \cdot \mathrm{min}^{-1}$. Sample temperature $(T)$ was controlled by an Optistat-DN cryostat (Oxford) connected to a model ITC601 $T$ controller. The MIR spectra of water sorbed into polymers were obtained by subtraction between the hydrated and dried samples. PMEA, PTHFA, and PMA of $10 \mathrm{mg}$ were mounted on a sapphire substrate with 13 mm diameter. The polymer was soaked in water for several minutes at room $T$ and then was removed from water and wiped with filter paper to remove excess water on their surfaces. Water in the polymer was slowly evaporated at ambient conditions while monitoring the weight of hydrated polymer by a CAP2P microbalance (Sartorius) until the sample weight approached water content $\left(\mathrm{W}_{\mathrm{C}}\right)$ expected. PMMA, which was prepared as a film on the substrate by a solvent-cast method, was hydrated by exposing them to humid air with $88 \%$ relative humidity $(\mathrm{RH})$ at $310 \mathrm{~K}$ for 10 days. The $\mathrm{W}_{\mathrm{C}}$ value of hydrated PMMA in weight percent was determined by the Karl Fischer (KF) method. The $\mathrm{W}_{\mathrm{C}}$ values for PMEA, PTHFA, PMA, and PMMA were 8.6, 4.8, 4.8, and $5.5 \mathrm{wt} \%$, respectively. The sample was set in a homemade sample-holder for the MIR measurement and then tightly sealed with indium.

2.3. Molecular Dynamics Simulation Molecular models employed in the present MD simulation to generate trajectories were the TIP4P2005 model ${ }^{17}$ for water and the general AMBER force field (GAFF) ${ }^{18}$ for the four polymers. The atomic partial charges for polymer molecules were generated in each monomer configuration using the restrained electrostatic potential (RESP) approach ${ }^{19}$ based on the electrostatic potential obtained at the B3LYP/6-31G (d) level using Gaussian $09^{20}$. Eight chains of 77 units each were initially contained in a cubic unit cell with water molecules. The number of water molecules was set to 414 for PMEA (8.5 wt \%), 148 for PMA (4.8 wt \%), 281 for PTHFA (5.0 wt $\%$ ) and 197 for PMMA (5.5 wt $\%$ ) systems, to be consistent with the experimental conditions. The PACKMOL package ${ }^{21}$ was employed to prepare initial configurations with random positions and orientations. To enhance statistical sampling, five independent MD trajectories starting from different initial configuration were prepared in parallel computations.

To remove inhomogeneous structures of the polymer systems in the initial configurations, some annealing procedures were carried out prior to statistical sampling for production. As the first annealing step, NPT ensemble simulation (constant number of molecules $N$, pressure $P=1$ atm and temperature $T=600 \mathrm{~K}$ ) was conducted during 5 ns, and the system was 
cooled down at the rate of $1.2 \times 10^{12} \mathrm{~K} \cdot \mathrm{min}^{-1}$ in a step by step manner. In these steps, the system was cooled to $100 \mathrm{~K}$ by steps of $50 \mathrm{~K}$ with $2.5 \mathrm{~ns}$ equilibration at each step in the NPT ensemble, and the specific volume was evaluated at the end of each equilibration to estimate $T_{\mathrm{g}}^{\mathrm{MD}}$ (see Sec. 3.2.1). After that, the final annealing step was conducted in the NPT ensemble during $1 \mathrm{~ns}$ at each target temperature, which was set in the range from $100 \mathrm{~K}$ to $300 \mathrm{~K}$ for PMEA system, from $100 \mathrm{~K}$ to $400 \mathrm{~K}$ for PMA and PTHFA systems, and from $100 \mathrm{~K}$ to $500 \mathrm{~K}$ for PMMA system in each $20 \mathrm{~K}$ interval. Here we define the time after the final annealing procedure as $t=0 \mathrm{~ns}$, and monitor time-development of the MD system for several tens of nano second in NVT ensemble because the MD systems may be still in a non-equilibrium state at $t=0 \mathrm{~ns}$. In Figure S1 of Supporting Information (SI), we show the result of H-bond states of water in the polymers (see Sec. 3.2.4.) averaged during (i) $t=0-1 \mathrm{~ns}$, (ii) $t=10-11 \mathrm{~ns}$, and (iii) $t=20-21 \mathrm{~ns}$, and confirmed that the result between (ii) and (iii) remain almost unchanged in comparison with those between (i) and (ii). Thus we mainly focus on the results averaged during (iii) in the present study. Here we notice that longer simulation may reach an equilibrium structure different from that in (iii). However, the results averaged during (iii) may be enough to find a relation between $T_{\mathrm{g}}^{\mathrm{MD}}$ and $T_{\mathrm{D}}^{\mathrm{MD}}$, and to observe a qualitative H-bonding states in the polymer systems.

In the above procedures, $T$ and $P$ were controlled by Nose-Hoover thermostat ${ }^{22,23}$ and Parrinello-Rahman barostat, ${ }^{24}$ respectively, with the time step of $1.0 \mathrm{fs}$. The intramolecular stretching vibrations of $\mathrm{H}$ atoms were constrained with the Lincs algorithm. ${ }^{25}$ Long-range electrostatic interactions were corrected with the particle-mesh Ewald method, ${ }^{26,27}$ and the cutoff length of the Lennard-Jones and the real space part of the Ewald sum were taken to be 1.2 nm. The GROMACS package (version 5.1) ${ }^{28}$ was employed in the present MD simulation.

\section{Results and Discussion \\ 3.1. Infrared spectra}

3.1.1. At $298 \mathrm{~K}$ MIR spectra of water sorbed into the four polymers and that of pure water at $298 \mathrm{~K}$ are given in Figure 2. Pure water has at least three components in its spectrum: two shoulders at $\sim 3650$ and $\sim 3240 \mathrm{~cm}^{-1}$ and a peak at $\sim 3400 \mathrm{~cm}^{-1}$. The four spectra of the sorbed water have at least four components at $\sim 3600, \sim 3530, \sim 3430$, and $\sim 3270 \mathrm{~cm}^{-1}$. The quite difference of the spectra between pure water and sorbed water indicates that most of the sorbed water interacts not only with water but also with the functional groups ( $\mathrm{O}$ atoms). The previous studies of $W_{\mathrm{C}}$-dependences of the spectrum of water sorbed into PMEA ${ }^{29,30}$ and polyethyleneglycol ${ }^{31,32}$ demonstrated that the relative absorbance of the two lower frequency components increases with $W_{\mathrm{C}}$. This suggests that the two lower and the two higher frequency components come from the $\mathrm{O}-\mathrm{H}$ groups $\mathrm{H}$-bonded to the $\mathrm{O}$ atom(s) of water molecule and of the polymers, respectively. The absorbance of the highest frequency component $\left(\sim 3600 \mathrm{~cm}^{-1}\right)$ may indicate the amount of water molecules $\mathrm{H}$-bonded to the $\mathrm{O}$ atoms in the polymers, and its absorbance is expressed hereafter as " $\mathrm{A}_{\mathrm{HYD}}$ ".

3.1.2. Temperature-dependence The spectra of the sorbed water in the temperature range of $298-170 \mathrm{~K}$ are given in the left two panels of Figure 3A-D, and those of pure water are given in Figure 3E. In the case of pure water, the spectra with a broad feature centered at $3400 \mathrm{~cm}^{-1}$, which is the characteristic peak for liquid water, suddenly change to the line shape with the sharp intense peak centered at $\sim 3250 \mathrm{~cm}^{-1}$, which is the characteristic peak for ice $I_{\mathrm{h}}$, at the temperature of $251 \mathrm{~K}$ during cooling. On the other hand, the ice spectra return to the liquid one at $273 \mathrm{~K}$ during rewarming. The temperature-dependence of absorbance at $3250 \mathrm{~cm}^{-1}\left(\mathrm{~A}_{3250}\right)$ is shown in the right panel of Figure 3 . The value of $\mathrm{A}_{3250}$ jumps up and down at $251 \mathrm{~K}$ during cooling and at $273 \mathrm{~K}$ during rewarming, respectively. In the case of water sorbed in the polymers, the temperature-dependence of the spectra is classified into the following three cases.

Case I (PMEA and PTHFA). Figures 3A and 3B show MIR response for the water-containing PMEA and PTHFA polymer systems, respectively. One can see that the ice peak at $\sim 3250 \mathrm{~cm}^{-1}$ appears during cooling, and further grows during rewarming, and disappears during subsequent rewarming. The former two enhancements are attributed to crystallization and recrystallization of the sorbed water, respectively, and the latter disappearance shows decrease of ice (sublimation). $T_{\mathrm{rc}}$ 's, in this case, are $215 \mathrm{~K}$ for PMEA and $222 \mathrm{~K}$ for PTHFA systems. The three transitions are invariably accompanied with the synchronous changes of $\mathrm{A}_{3250}$ and $\mathrm{A}_{\mathrm{HYD}}$, suggesting that the water molecules binding to the $\mathrm{O}$ atoms in the polymers participate in the three transitions.

One can also see from the right panels of Figures 3A and 3B that the amount of crystallization during cooling strongly depends on the cooling rate: the $\mathrm{A}_{3250}$ value gradually increases from $227 \mathrm{~K}$ to $208 \mathrm{~K}$ for PMEA and from $242 \mathrm{~K}$ to $217 \mathrm{~K}$ for PTHFA at the cooling rate of $0.5 \mathrm{~K} \cdot \mathrm{min}^{-1}$, whereas no crystallization is observed at $4.8 \mathrm{~K} \cdot \mathrm{min}^{-1}$. This gradual increase of $\mathrm{A}_{3250}$ during cooling is remarkably different from the sudden increase in the case of pure water (Figure 3E). This difference demonstrates that the crystallization of pure water takes place via reorientation of liquid water molecules, while crystallization of the sorbed water is caused by deposition of water after diffusion of monomolecular water sorbed into the polymer matrix. One can also see that the recrystallization occurs in the wide temperature range of 215-235 K for PMEA and of 222-250 K for PTHFA, suggesting the recrystallization originates from the diffusion mechanism of water molecules trapped on the polymer chains rather than the reorientation mechanism.

Case II (PMA). In PMA system, crystallization of the sorbed water at $255 \mathrm{~K}$ with the additional second jump-up at $234 \mathrm{~K}$ during cooling and recrystallization at $225 \mathrm{~K}$ are observed. The difference spectrum of MIR spectra at the temperatures $240 \mathrm{~K}$ and $260 \mathrm{~K}$ (see Figure S2 in SI) shows the gradual increase of $\mathrm{A}_{3250}$ in the temperature range of 
255-234 $\mathrm{K}$ is due to crystallization of the sorbed water. The jump-up at $234 \mathrm{~K}$ can be explained by the crystallization of liquid water, which is formed by water monomer, because the homogeneous nucleation temperature of pure water is reported to be $236 \mathrm{~K} .^{33-36}$

Case III (PMMA). In both cooling and rewarming processes, the characteristic peak of ice $I_{\mathrm{h}}$ in the spectra is almost missing in the whole temperature range, and any steep changes in $\mathrm{A}_{3250}$ and $\mathrm{A}_{\mathrm{HYD}}$ are hardly observed. These results suggest that the transitions for the sorbed water rarely take place. This may be due to the fact that the transition of water diffusion for PMMA, which is a trigger of the crystallization/recrystallization as discussed below, occurs at the temperature higher than the melting point of water, $273.15 \mathrm{~K}$, indicating the ice formation is not possible for the PMMA system.

3.1.3. Correlation of $\boldsymbol{T}_{\mathrm{rc}}$ and $\boldsymbol{T}_{\mathrm{g}}^{\mathrm{DSC}}$ In Figure 4, the recrystallization temperature measured by the MIR spectroscopy, $T_{\mathrm{rc}}$, versus the glass transition temperature of the polymers reported previously, ${ }^{10,11,37,38} T_{\mathrm{g}}^{\mathrm{DSC}}$, are plotted except for PMMA system. Note that $T_{\mathrm{rc}}$ for PMMA was hardly visible in Figure 3D. A positive correlation between these temperatures is clearly seen. The result also shows $T_{\mathrm{g}}^{\mathrm{DSC}}$ is always larger than $T_{\mathrm{rc}}$. The difference of $T_{\mathrm{g}}^{\mathrm{DSC}}$ and $T_{\mathrm{rc}}$ may be caused by the fact that $T_{\mathrm{rc}}$ is a measure of the transition for water interacting with polymer, while $T_{\mathrm{g}}^{\mathrm{DSC}}$ is the transition of mobility of polymer itself. The relation of $T_{\mathrm{g}}^{\mathrm{DSC}}>T_{\mathrm{rc}}$ suggests that the recrystallization phenomenon, which occurs in diffusion-controlled process, is a precursor of the glass transition of polymer. This finding is also supported by the present MD simulation, in which the glass transition temperature is always larger than the lower limit temperature of the diffusion of water in the polymers matrices, $T_{\mathrm{g}}^{\mathrm{MD}}>T_{\mathrm{D}}^{\mathrm{MD}}$, as discussed in Sec. 3.2.3.

In addition to the recrystallization temperature, the ending temperatures of crystallization during cooling, referred to as crystallization temperatures (208 K for PMEA, $217 \mathrm{~K}$ for PTHFA, $222 \mathrm{~K}$ for PMA in Figure 3), are plotted against $T_{\mathrm{g}}^{\mathrm{DSC}}$ in Figure 4. Not only $T_{\text {rc }}$ 's but also the ending temperatures of crystallization are positively correlated with $T_{\mathrm{g}}^{\mathrm{DSC}}$, and hence the ending temperatures of crystallization and the onset temperature of recrystallization are positively correlated and similar to each other. These temperatures are also close to the transition temperature of water diffusion, $T_{\mathrm{D}}^{\mathrm{MD}}$, as discussed in Sec.3.2.2, suggesting that the crystallization of water in the present polymer systems is also driven by the diffusion mechanism rather than the reorientation mechanism. The diffusion mechanism of the crystallization is also supported by the result of MIR spectra (see the right panel of Figure 3), where the increase of $A_{3250}$ and the decrease of $A_{H Y D}$ are taken place simultaneously, indicating the water molecules interacting with polymer are detached (i.e., the decrease of $\mathrm{A}_{\mathrm{HYD}}$ ), and then condense and crystallize (i.e., the increase of $\mathrm{A}_{3250}$ ) after diffusing in the polymer matrix. If the reorientation mechanism was valid, the decrease of $A_{H Y D}$ would not be observed when $A_{3250}$ increases.

\subsection{Molecular dynamics simulation}

3.2.1. Calculation of $T_{\mathbf{g}}^{\mathrm{MD}} \quad$ In a NPT ensemble MD simulation, cell box size is automatically adjusted in a given temperature and pressure. Thus we can obtain specific volume of the present polymer systems, $V_{\mathrm{SP}}^{\mathrm{MD}}$, as a function of temperature at $P=1 \mathrm{~atm}$. In Figure $5, V_{\mathrm{SP}}^{\mathrm{MD}}$ 's are plotted against the system temperature. One can see $V_{\mathrm{SP}}^{\mathrm{MD}}$ 's increases with increasing the temperature due to thermal expansion. In all cases, a transition of the gradient for $V_{\mathrm{SP}}^{\mathrm{MD}}$-temperature plot can be seen at a temperature characteristic to each polymer system. This indicates that the thermal expansion coefficient abruptly changes at this temperature, which is widely accepted as a glass transition temperature in MD system ${ }^{39-41}, T_{\mathrm{g}}^{\mathrm{MD} *}$. The calculated $T_{\mathrm{g}}^{\mathrm{MD} *}$, s are $337 \mathrm{~K}$ for PMEA, $370 \mathrm{~K}$ for PTHFA, $375 \mathrm{~K}$ for PMA, and $423 \mathrm{~K}$ for PMMA, which tend to be much higher than the experimental $T_{\mathrm{g}}^{\mathrm{DSC}}$ 's: $223 \mathrm{~K}$ for $\mathrm{PMEA}^{10}, 260 \mathrm{~K}$ for PTHFA ${ }^{11}, 291 \mathrm{~K}$ for $\mathrm{PMA}^{38}$, and $381 \mathrm{~K}$ for $\mathrm{PMMA}^{37}$. This is because $T_{\mathrm{g}}$ usually depends on the cooling rate $q_{\mathrm{g}}$, and $q_{\mathrm{g}}$ in an annealing procedure for MD system is much larger than that of the experimental conditions. To compare a glass transition temperature obtained in MD simulation with that in experiment, a correction of $T_{\mathrm{g}}^{\mathrm{MD} *}$ should be considered in the following way. The MD study by Soldera and Metatla showed that $T_{\mathrm{g}}^{\mathrm{MD*}} \mathrm{s}$ for PMA and PMMA are $\sim 100 \mathrm{~K}$ higher than the experimental values, ${ }^{42-44}$ which is rationalized by the Williams-Landel-Ferry equation written as ${ }^{43,45}$

$$
\Delta T_{\mathrm{g}}=\frac{-B \log _{10} \frac{q_{\mathrm{g}, 2}}{q_{\mathrm{g}, 1}}}{A+\log _{10} \frac{q_{\mathrm{g}, 2}}{q_{\mathrm{g}, 1}}}
$$

where $\Delta T_{\mathrm{g}}=T_{\mathrm{g}, 1}-T_{\mathrm{g}, 2}$ is the difference of the glass transition temperatures between $T_{\mathrm{g}, 1}$ at the cooling rate $q_{\mathrm{g}, 1}$ and $T_{\mathrm{g}, 2}$ at the cooling rate $q_{\mathrm{g}, 2} . A$ and $B$ are the fitting parameters. In Ref. 43, $T_{\mathrm{g}, n}$ and $q_{\mathrm{g}, n}$ in MD simulation $(n=1)$ and in experiment $(n=2)$ were employed, and $A=16.7$ and $B=48 \mathrm{~K}$ were obtained as average values for some polymer systems. Substituting the cooling rate in the present MD simulation $q_{\mathrm{g}, 1}=1.2 \times 10^{12} \mathrm{~K} \cdot \mathrm{min}^{-1}$ and in experiment $q_{\mathrm{g}, 2}=2.5$ $\mathrm{K} \cdot \mathrm{min}^{-1}$ for $\mathrm{PMEA}^{10}, 3.0 \mathrm{~K} \cdot \mathrm{min}^{-1}$ for PTHFA ${ }^{11}, 40 \mathrm{~K} \cdot \mathrm{min}^{-1}$ for $\mathrm{PMA}^{38}$, and $10 \mathrm{~K} \cdot \mathrm{min}^{-1}$ for PMMA ${ }^{37}$ into eq. 1 , we have $\Delta T_{\mathrm{g}}=112 \mathrm{~K}$ for PMEA, $109 \mathrm{~K}$ for PTHFA, $80.8 \mathrm{~K}$ for PMA, and $94.6 \mathrm{~K}$ for PMMA. The corrected glass transition temperature in MD simulation, $T_{\mathrm{g}}^{\mathrm{MD}}=T_{\mathrm{g}}^{\mathrm{MD} *}-\Delta T_{\mathrm{g}}$, to compare with the experimental value are $225 \mathrm{~K}$ for PMEA, $261 \mathrm{~K}$ for PTHFA, $294 \mathrm{~K}$ for PMA, and $328 \mathrm{~K}$ for PMMA, which are in satisfactory agreement with the experimentally reported glass transition temperatures indicated above. 
3.2.2. Calculation of diffusion coefficient and $T_{\mathrm{D}}^{\mathrm{MD}}$ The diffusion coefficient, $D$, of the sorbed water was calculated from mean-squared displacement of molecule in each system according to the Einstein formula: ${ }^{46}$

$$
D=\lim _{t \rightarrow \infty} \frac{1}{6 t}\left\langle\frac{1}{N} \sum_{i}^{N}\left|\boldsymbol{r}_{i}(t)-\boldsymbol{r}_{i}(0)\right|^{2}\right\rangle
$$

where $N$ is the number of molecule in MD system, and $\boldsymbol{r}_{i}(t)$ is the center-of-mass position of the ith molecule at time $t$. In Figure 6, the calculated diffusion coefficients of water in each polymer system are shown in an Arrhenius plot. The $\log D$ values decrease linearly against temperature and has a transition at a certain temperature, defined as $T_{\mathrm{D}}^{\mathrm{MD}}$. In all cases, the $D$ values below $T_{\mathrm{D}}^{\mathrm{MD}}$ have $10^{-11}-10^{-12} \mathrm{~m}^{2} \cdot \mathrm{s}^{-1}$, which is much smaller than the values of pure liquid water, $\sim 10^{-9} \mathrm{~m}^{2} \cdot \mathrm{s}^{-1}$. Thus the sorbed water molecules may tend to be fixed and thermally vibrate near the $\mathrm{O}$ atoms of the polymer in the lower temperature range. In the higher temperature range, on the other hand, the sorbed water molecules actually diffuse in the polymer matrix ${ }^{47}$. In this sense, $T_{\mathrm{D}}^{\mathrm{MD}}$ can be considered to be the lower limit temperature of the diffusion for the sorbed water. The present result shows $T_{\mathrm{D}}^{\mathrm{MD}}$ has $203 \mathrm{~K}$ for PMEA, $231 \mathrm{~K}$ for PTHFA, $254 \mathrm{~K}$ for PMA, and $300 \mathrm{~K}$ for PMMA. Chen et al. also calculated diffusion coefficient of water in an amorphous polyacrylate latex film by MD simulation, and reported the similar temperature dependence of $D$ values ${ }^{48}$.

3.2.3. Correlation of $\boldsymbol{T}_{\mathrm{D}}^{\mathrm{MD}}$ and $\boldsymbol{T}_{\mathrm{g}}^{\mathrm{MD}}$ In Figure $7, T_{\mathrm{D}}^{\mathrm{MD}}$ against $T_{\mathrm{g}}^{\mathrm{MD}}$ obtained in the present $\mathrm{MD}$ simulation are shown for each polymer. Similar to the experimental result of $T_{\mathrm{rc}}$ and $T_{\mathrm{g}}^{\mathrm{DSC}}, T_{\mathrm{D}}^{\mathrm{MD}}$ and $T_{\mathrm{g}}^{\mathrm{MD}}$ have a positive correlation. This correlation suggests that the onset temperature of diffusion at low temperature is related to mobility of the polymers. Chen $e t$ al. discussed that the diffusion rate depends on the local chain mobility and the dissociation of water molecules from the polar groups of the polymers ${ }^{48}$. In particular, $T_{\mathrm{g}}^{\mathrm{MD}}>T_{\mathrm{D}}^{\mathrm{MD}}$ holds, indicating that the lower limit temperature of water diffusion is a precursor of the local mobility of polymers. In the present systems, water molecule is much smaller (or shorter) than the side chain of polymer, and hence it can be expected that the transition of mobility of water, that is $T_{\mathrm{D}}^{\mathrm{MD}}$, appears at the temperature lower than the glass transition temperature of each polymer. One can also speculate that polymers may make some spaces to enhance the water diffusion at a temperature slightly below the glass transition temperature. Further research may be needed to account for physical origin of the relation $T_{\mathrm{g}}^{\mathrm{MD}}>T_{\mathrm{D}}^{\mathrm{MD}}$.

Combining the correlation relation of $T_{\mathrm{D}}^{\mathrm{MD}}-T_{\mathrm{g}}^{\mathrm{MD}}$ and that of $T_{\mathrm{rc}}-T_{\mathrm{g}}^{\mathrm{DSC}}$ discussed in Sec.3.1.3, we can conclude that the recrystallization temperature is strongly correlated with the water diffusion in the polymers, strongly supporting the recrystallization process is raised by the diffusion mechanism rather than the reorientation mechanism.

3.2.4. Hydrogen bonding states of water Here we discuss $\mathrm{H}$-bonding states of water molecules in the polymers as a function of temperature. The states considered here are illustrated in Figure 1, where solid and dotted lines show the covalent bond and $\mathrm{H}$-bond, respectively. Arabic number (1-4) on $\mathrm{O}-\mathrm{H}$ bond indicates the category of $\mathrm{O}-\mathrm{H}$ group of water molecules: O-H(1) represents the state bonding to oxygen atom of water $(\mathrm{O}),(2)$ to carbonyl $\mathrm{O}$, and (3) to ether O's of each polymers. Otherwise, a state is categorized in $\mathrm{O}-\mathrm{H}(4)$, not $\mathrm{H}$-bonded to any $\mathrm{O}$ 's (free $\mathrm{O}-\mathrm{H}$ ). If the distance between $\mathrm{H}$ atom of water molecule and $\mathrm{O}$ atom of each functional group is less than $2.5 \AA^{49,50}$, the $\mathrm{O}-\mathrm{H} \cdots \mathrm{O}$ group is defined to be $\mathrm{H}$-bonded.

Figure 8 shows ratio of the four $\mathrm{H}$-bonding states as a function of temperature for each polymer system. In all of the systems, the free $\mathrm{O}-\mathrm{H}$ group (filled circle, $\mathrm{O}-\mathrm{H}(4)$ ) is nearly zero at the lowest temperature, indicating that almost all the water molecules bonds to oxygen sites at the temperature. The ratio of $\mathrm{O}-\mathrm{H}(4)$ rapidly increases with increasing temperature over $\sim 210 \mathrm{~K}$ in PMEA, $220 \mathrm{~K}$ in PTHFA, $230 \mathrm{~K}$ in PMA, and $\sim 300 \mathrm{~K}$ in PMMA, which are very close to $T_{\mathrm{D}}^{\mathrm{MD}}$ 's. On the other hand, the ratio of the $\mathrm{O}-\mathrm{H}$ group bonding to the carbonyl $\mathrm{O}$ atom (triangle, $\mathrm{O}-\mathrm{H}(2)$ ) is $40-65 \%$ at the lowest temperature and suddenly decreases at the temperature near $T_{\mathrm{D}}^{\mathrm{MD}}$. The ratio of the $\mathrm{O}-\mathrm{H}$ group bonding to ether $\mathrm{O}$ atoms (open circle, $\mathrm{O}-\mathrm{H}(3)$ ) is $2-3 \%$ in PMMA and PMA and 10-15\% in PMEA and PTHFA at the lowest temperature, and decreases near $T_{\mathrm{D}}^{\mathrm{MD}}$ especially for PMEA and PTHFA systems. The very small ratio of $\mathrm{O}-\mathrm{H}(3)$ group compared with that of $\mathrm{O}-\mathrm{H}(2)$ indicates that the sorbed water almost bonds to the carbonyl $\mathrm{O}$ atoms. The ratio of the $\mathrm{O}-\mathrm{H}$ group bonding to water $\mathrm{O}$ atom (cross, $\mathrm{O}-\mathrm{H}(1)$ ) is $30-50 \%$ and suddenly increases near $T_{\mathrm{D}}^{\mathrm{MD}}$ especially for PMEA and PTHFA systems. These results strongly support the recrystallization is driven by the diffusion-controlled process. Our MD simulation unveils the detailed picture of the diffusion-controlled process for recrystallization during rewarming: At the temperature lower than $T_{\mathrm{D}}^{\mathrm{MD}}$, major part of $\mathrm{O}-\mathrm{H}$ groups bonds with the carbonyl groups or water. In the case of PMEA and PTHFA systems, the $\mathrm{O}-\mathrm{H}$ 's bonded with the carbonyl groups start to be detached over $T_{\mathrm{D}}^{\mathrm{MD}}$, and then they condense into a cluster after diffusing in the polymer matrix. The condensed water cluster crystallizes as long as the temperature is lower than the melting point of pure water, $T_{\mathrm{m}}$. In the case of PMMA, on the other hand, $T_{\mathrm{D}}^{\mathrm{MD}}$ is near or over $T_{\mathrm{m}}$, resulting in no observation of recrystallization.

The diffusion mechanism is also supported by the following MD simulation result: in Figure S3 and S4 of SI, one can see more than $90 \%$ of water clusters formed in the PMEA polymer at $100 \mathrm{~K}$ consists of the number of water molecules less than 20. Pradzynski et al. ${ }^{51}$ reported that an appearance of the characteristic IR peak at $\sim 3200 \mathrm{~cm}^{-1}$ for ice $I_{\mathrm{h}}$, which is observed in the present polymer systems, needed water cluster constituting at least $\sim 475$ water molecules. If the reorientation mechanism was valid, the present polymer systems could contain such large clusters. 


\section{Conclusion}

Recrystallization behavior of water sorbed into the four poly(meth)acrylates is investigated by VT-MIR spectroscopy and MD simulation. VT-MIR spectra showed a positive correlation between $T_{\mathrm{rc}}$ and $T_{\mathrm{g}}^{\mathrm{DSC}}$ for PMEA, PTHFA, and PMA systems, suggesting the recrystallization is related to a mobility of the polymers. MD simulation, on the other hand, showed a positive correlation between $T_{\mathrm{D}}^{\mathrm{MD}}$ and $T_{\mathrm{g}}^{\mathrm{MD}}$ for all the polymers treated here. These results demonstrate the recrystallization takes place by the diffusion mechanism rather than the reorientation mechanism. No observation of the recrystallization for PMMA system originates from the fact that $T_{\mathrm{D}}^{\mathrm{MD}}$ of it is near or over the melting point temperature of pure water. Not abrupt but gradual change of the ice peak as a function of temperature in the VT-MIR spectra also elucidates not only recrystallization but also crystallization is dominated by the diffusion-controlled process. The analysis of the $\mathrm{H}$-bond states in the polymers by MD simulation illustrates the water molecules bonded with the carbonyl oxygens are detached over the recrystallization temperature, and the subsequent diffusion process enhances the formation of water cluster, resulting in the observation of recrystallization.

Acknowledgements. The authors are indebted to T. Motonaga and S. Kishinaka for their technical assistance. The MD calculations were performed using the supercomputers at Research Center for Computational Science, Okazaki, Japan. This work was supported by the Japan Society for the Promotion of Science (JSPS) KAKENHI Grant Numbers 15K04604 and $16 \mathrm{H} 04095$.

Supporting Information Available. Temperature dependence of the hydrogen bonding states of the sorbed water at various calculation times of $0-1 \mathrm{~ns}, 10-11 \mathrm{~ns}$, and also $20-21 \mathrm{~ns}$, subtracted spectrum of the sorbed water in PMA at $240 \mathrm{~K}$ and $260 \mathrm{~K}$, water cluster size distribution contained in the present PMEA system, and a snapshot of water cluster in the polymer. This material is available free of charge via the Internet at http://pubs.acs.org.

\section{References}

(1) Lynden-Bel, R. M.; Morris, S. C.; Barrow, J. D.; Finney, J. L.; Harper, J. C. L. . Water and Life: The Unique Properties of H2O; Taylor \& Francis Publishe (CRC Press): London, 2010.

(2) Brüggeller, P.; Mayer, E. Complete Vitrification in Pure Liquid Water and Dilute Aqueous Solutions. Nature 1980, 288, 569-571.

(3) Mayer, E.; Brüggeller, P. Vitrification of Pure Liquid Water by High Pressure Jet Freezing. Nature 1982, 298, 715718.

(4) Nishinari, K.; Watase, M.; Hatakeyama, T. Effects of Polyols and Sugars on the Structure of Water in Concentrated Gelatin Gels as Studied by Low Temperature Differential Scanning Calorimetry. Colloid Polym. Sci. 1997, 275 (11), $1078-1082$.

(5) Hatakeyama, H.; Hatakeyama, T. Interaction between Water and Hydrophilic Polymers. Thermochim. Acta 1998, $308(1-2), 3-22$.

(6) Huang, L.; Nishinari, K. Interaction between Poly(ethylene Glycol) and Water as Studied by Differential Scanning Calorimetry. J. Polym. Sci. Part B Polym. Phys. 2001, 39 (5), 496-506.

(7) Tanaka, M.; Mochizuki, A. Effect of Water Structure on Blood Compatibility--Thermal Analysis of Water in Poly(meth)acrylate. J. Biomed. Mater. Res. A 2004, 68 (4), 684-695.

(8) Hatakeyma, T.; Kasuga, H.; Tanaka, M.; Hatakeyama, H. Cold Crystallization of Poly(ethylene Glycol)-Water Systems. Thermochim. Acta 2007, 465 (1-2), 59-66.

(9) Furushima, Y.; Ishikiriyama, K.; Ueno, Y.; Sugaya, H. Analysis of the State of Water in Polyvinylpyrrolidone Aqueous Solutions Using DSC Method. Thermochim. Acta 2012, 538, $43-47$.

(10) Tanaka, M.; Motomura, T.; Ishii, N.; Shimura, K.; Onishi, M.; Mochizuki, A.; Hatakeyama, T. Cold Crystallization of Water in Hydrated poly(2-Methoxyethyl Acrylate) (PMEA). Polym. Int. 2000, 49 (12), 1709-1713.

(11) Mochizuki, A.; Hatakeyama, T.; Tomono, Y.; Tanaka, M. Water Structure and Blood Compatibility of Poly(tetrahydrofurfuryl Acrylate). J. Biomater. Sci. Polym. Ed. 2009, 20 (5-6), 591-603.

(12) Gemmei-Ide, M.; Kitano, H. Recrystallization of Water in a Non-Water-Soluble Polymer Examined by Fourier Transform Infrared Spectroscopy: Poly(2-Methoxyethylacrylate) with Low Water Content. J. Phys. Chem. B 2008, $112(41), 12863-12866$.

(13) Gemmei-Ide, M.; Kitano, H. Spectroscopic Evidence of Phase Transition of Monomolecular Water in Solid 
Polystyrene. J. Phys. Chem. B 2008, 112 (10), 2764-2766.

(14) Gemmei-Ide, M.; Ohya, A.; Kitano, H. Recrystallization of Water in Non-Water-Soluble (Meth)acrylate Polymers Is Not Rare and Is Not Devitrification. J. Phys. Chem. B 2012, 116 (6), 1850-1857.

(15) Gemmei-Ide, M.; Kitano, H. Crystal Growth of Ice Ih by Revapor-Deposition and Diffusion Suppression of Monomolecular Water in a Polymer Solid: Spectroscopic Observation of Phase Transition of Water Sorbed into Solid Polystyrene. J. Phys. Chem. B 2008, 112 (43), 13499-13502.

(16) Lee, W. J.; Chang, J. G.; Ju, S. P. Hydrogen-Bond Structure at the Interfaces between Water/poly(methyl Methacrylate), Water/poly(methacrylic Acid), and water/poly(2- Aminoethylmethacrylamide). Langmuir 2010, 26 (15), 12640-12647.

(17) Abascal, J. L.; Vega, C. A General Purpose Model for the Condensed Phases of Water: TIP4P/2005. J. Chem. Phys. 2005, $123(23), 234505$.

(18) Wang, J. M.; Wolf, R. M.; Caldwell, J. W.; Kollman, P. a; Case, D. a. Development and Testing of a General Amber Force Field. J. Comput. Chem. 2004, 25 (9), 1157-1174.

(19) Bayly, C. I.; Cieplak, P.; Cornell, W.; Kollman, P. A. A Well-Behaved Electrostatic Potential Based Method Using Charge Restraints for Deriving Atomic Charges: The RESP Model. J. Phys. Chem. 1993, 97, 10269-10280.

(20) Frisch, M. J.; Trucks, G. W.; Schlegel, H. B.; Scuseria, G. E.; Robb, M. A.; Cheeseman, J. R.; Scalmani, G.; Barone, V.; Mennucci B.; Petersson, G. A. ; et al. Gaussian 09, Revision A.02; Gaussian, Inc.: Wallingford CT, 2009.

(21) Martinez, L.; Andrade, R.; Birgin, E. G.; Martinez, J. M. PACKMOL: A Package for Building Initial Configurations for Molecular Dynamics Simulations. J. Comput. Chem. 2009, 30, 2157-2164.

(22) Hoover, W. G. Canonical Dynamics: Equilibrium Phase-Space Distributions. Phys. Rev. A 1985, 31 (3), $1695-1697$.

(23) Nosé, S. A Unified Formulation of the Constant Temperature Molecular Dynamics Methods. J. Chem. Phys. 1984, $81(1), 511-519$.

(24) Parrinello, M.; Rahman, A. Polymorphic Transitions in Single Crystals: A New Molecular Dynamics Method. $J$. Appl. Phys. 1981, 52 (12), 7182-7190.

(25) Hess, B.; Bekker, H.; Berendsen, H. J. C.; Fraaije, J. G. E. M. LINCS: A Linear Constraint Solver for Molecular Simulations. J. Comput. Chem. 1997, 18 (12), 1463-1472.

(26) Essmann, U.; Perera, L.; Berkowitz, M. L.; Darden, T.; Lee, H.; Pedersen, L. G. A Smooth Particle Mesh Ewald Method. J Chem Phys 1995, 103 (1995), 8577-8593.

(27) Darden, T.; York, D.; Pedersen, L. Particle Mesh Ewald: An N· $\log (\mathrm{N})$ Method for Ewald Sums in Large Systems. J. Chem. Phys. 1993, 98 (12), 10089.

(28) Pronk, S.; Páll, S.; Schulz, R.; Larsson, P.; Bjelkmar, P.; Apostolov, R.; Shirts, M. R.; Smith, J. C.; Kasson, P. M.; Van Der Spoel, D.; et al. GROMACS 4.5: A High-Throughput and Highly Parallel Open Source Molecular Simulation Toolkit. Bioinformatics 2013, 29 (7), 845-854.

(29) Ide, M.; Mori, T.; Ichikawa, K.; Kitano, H.; Tanaka, M.; Mochizuki, A.; Oshiyama, H.; Mizuno, W. Structure of Water Sorbed into poly(MEA-Co-HEMA) Films as Examined by ATR-IR Spectroscopy. Langmuir 2003, 19 (2), 429-435.

(30) Morita, S.; Tanaka, M.; Ozaki, Y. Time-Resolved in Situ ATR-IR Observations of the Process of Sorption of Water into a poly(2-Methoxyethyl Acrylate) Film. Langmuir 2007, 23 (7), 3750-3761.

(31) Ide, M.; Yoshikawa, D.; Maeda, Y.; Kitano, H. State of Water inside and at the Surface of Poly(ethylene Glycol) Films Examined by FT-IRâ€ . Langmuir 1999, 15 (4), 926-929.

(32) Gemmei-Ide, M.; Motonaga, T.; Kitano, H. State of Irremovable Water in Solid Polymer Films Examined by Fourier Transform Infrared Spectroscopy I: Poly(ethylene Glycol) Dimethyl Ether. Langmuir 2006, 22 (6), $2422-$ 2425.

(33) Hobbs, P. V. Ice Physics; Clarendon Press: Oxford, U.K., 1974; pp 461-523.

(34) Franks, F. Water: A Comprehensive Treatise; Franks, F., Ed.; New York, 1982; pp 1-81.

(35) Angell, C. A.; Rodgers, V. Near Infrared Spectra and the Disrupted Network Model of Normal and Supercooled 
Water. J. Chem. Phys. 1984, 80 (15), 6245-.

(36) Mishima, O.; Stanley, H. E. The Relationship between Liquid, Supercooled and Glassy Water. Nature 1998, 396 (6709), 329-335.

(37) Huang, Y.; Paul, D. R. Effect of MolecularWeight and Temperature on Physical Aging of ThinGlassy Poly(2,6-Dimethyl-1,4-Phenylene Oxide) Films. J. Polym. Sci. Part B Polym. Phys. 2007, 45 (April), 1390-1398.

(38) Gomez-Ribelles, J. L.; Monleon Pradas, M.; Gallego Ferrer, G.; Peidro Torres, N.; Perez Gimenez, V.; Pissis, P.; Kyritsis, A. Poly ( Methyl Acrylate )/ Poly ( Hydroxyethyl Acrylate ) Sequential Interpenetrating Polymer Networks . Miscibility and Water Sorption Behavior. J. Polym. Sci. Part B Polym. Phys. 1999, 37, 1587-1599.

(39) Han, J.; Gee, R. H.; Boyd, R. H. Glass Transition Temperatures of Polymers from Molecular Dynamics Simulations. Macromolecules 1994, 27, 7781-7784.

(40) Roe, R. J. Molecular Dynamics Simulation Study of Short Time Dynamics in Polystyrene. J. Non. Cryst. Solids 1998, 235-237, 308-313.

(41) Plante, A.; Palato, S.; Lebel, O.; Soldera, A. Functionalization of Molecular Glasses: Effect on the Glass Transition Temperature. J. Mater. Chem. C 2013, 1 (5), 1037.

(42) Soldera, A.; Metatla, N. Glass Transition Phenomena Observed in Stereoregular PMMAs Using Molecular Modeling. Compos. Part A Appl. Sci. Manuf. 2005, 36 (4), 521-530.

(43) Soldera, A.; Metatla, N. Glass Transition of Polymers: Atomistic Simulation versus Experiments. Phys. Rev. E - Stat. Nonlinear, Soft Matter Phys. 2006, 74 (6), 1-6.

(44) Soldera, A. Atomistic Simulations of Vinyl Polymers. Mol. Simul. 2012, 38 (8-9), 762-771.

(45) Ozmaian, M.; Naghdabadi, R. Molecular Dynamics Simulation Study of Glass Transition in Hydrated Nafion. $J$. Polym. Sci. Part B Polym. Phys. 2014, 52 (13), 907-915.

(46) Allen, M. P.; Tildesley, D. J. Computer Simulation of Liquids; Clarendon: Oxford, U.K, 1987.

(47) Sheikholeslam, S. A.; Manzano, H.; Grecu, C.; Ivanov, A. Reduced Hydrogen Diffusion in Strained Amorphous SiO2: Understanding Ageing in MOSFET Devices. J. Mater. Chem. C 2016, 4 (34), 8104-8110.

(48) Chen, Z.; Gu, Q.; Zou, H.; Zhao, T.; Wang, H. Molecular Dynamics Simulation of Water Diffusion inside an Amorphous Polyacrylate Latex Film. J. Polym. Sci. Part B Polym. Phys. 2007, 45 (April), 884-891.

(49) Lin, J.-H.; Baumgaertner, A. Molecular Dynamics Simulations of Hydrophobic and Amphipatic Proteis Interacting with a Lipid Bilayer Membrane. Comput. Theor.¥ Poly.¥ Sci. 2000, 10, 97-102.

(50) Kotelyanskii, M. J.; Wagner, N. J.; Paulaitis, M. E. Molecular Dynamics Simulation Study of the Mechanisms of Water Diffusion in a Hydrated, Amorphous Polyamide. Comput. Theor. Polym. Sci. 1999, 9 (3-4), 301-306.

(51) Pradzynski, C. C.; Forck, R. M.; Zeuch, T.; Slavicek, P.; Buck, U. A Fully Size-Resolved Perspective on the Crystallization of Water Clusters. Science (80-. ). 2012, 337 (6101), 1529-1532. 


\section{TOC graphic}

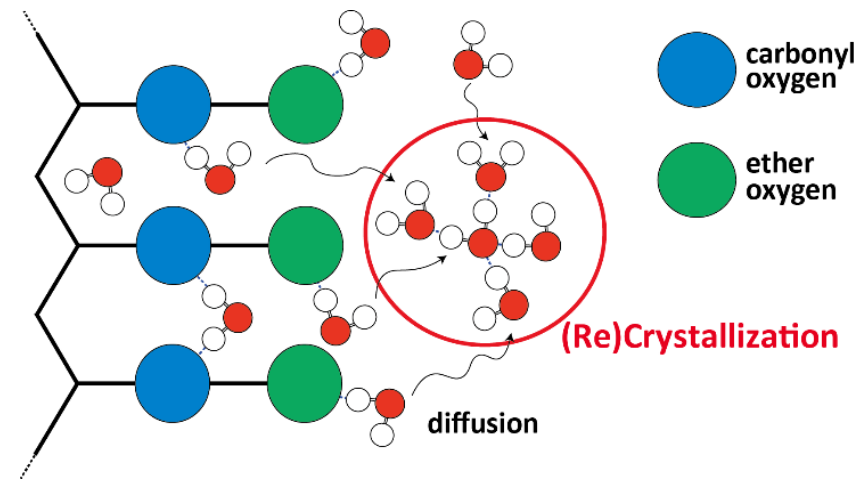

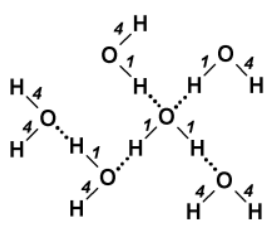

Water molecule

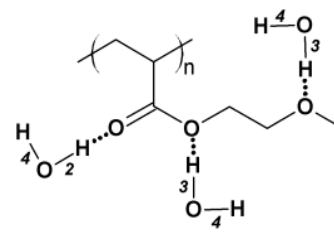

PMEA

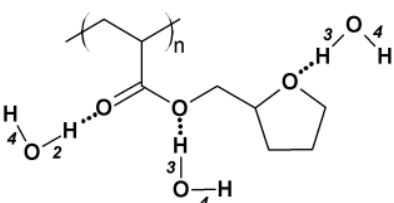

PTHFA<smiles>[Y]C(CC)C(=O)C(=O)OC</smiles>

PMA

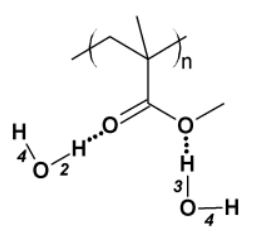

PMMA

Figure 1. Chemical structures of the polymers and possible hydrogen bonding states between water molecule and polymers. Arabic number (1-4) on $\mathrm{O}-\mathrm{H}$ bond indicates the category of $\mathrm{O}-\mathrm{H}$ group of water based on hydrogen bonding state. $\mathrm{O}-\mathrm{H}(1)$ indicates the state bonding to water oxygen atom (O), (2) to carbonyl $\mathrm{O}$, and (3) to ether $\mathrm{O} . \mathrm{O}-\mathrm{H}(4)$ indicates the no bonding state (free $\mathrm{O}-\mathrm{H}$ ).

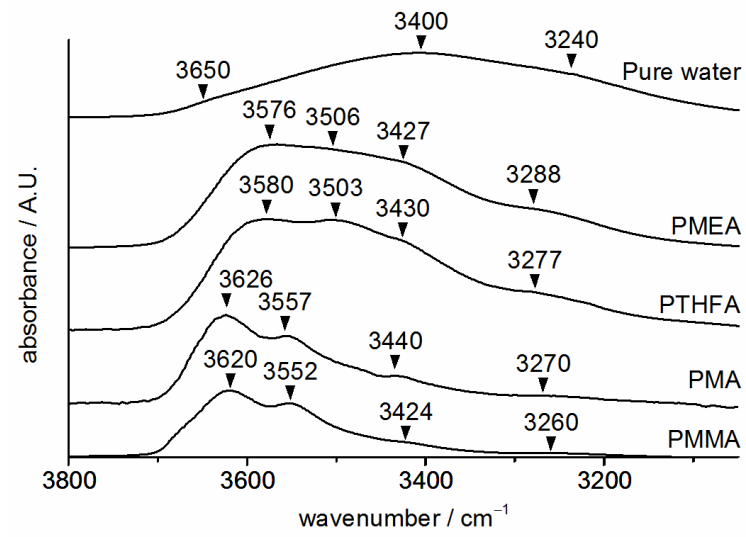

Figure 2. MIR spectra of water sorbed into PMEA with $\mathrm{W}_{\mathrm{C}}$ of $8.6 \mathrm{wt} \%$, PTHFA with $\mathrm{W}_{\mathrm{C}}$ of $4.8 \mathrm{wt} \%$, PMA with $\mathrm{W}_{\mathrm{C}}$ of $4.8 \mathrm{wt} \%$, and PMMA with $\mathrm{W}_{\mathrm{C}}$ of $5.5 \mathrm{wt} \%$, and pure water at $298 \mathrm{~K}$. 
(A) PMEA

Cooling process

Heating process

Cooling process

Heating process
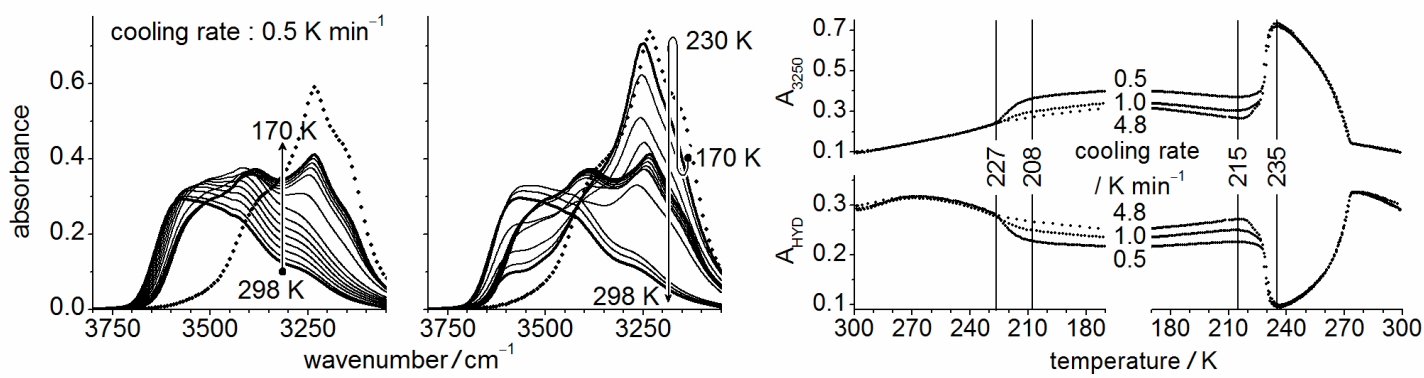

(B) PTHFA
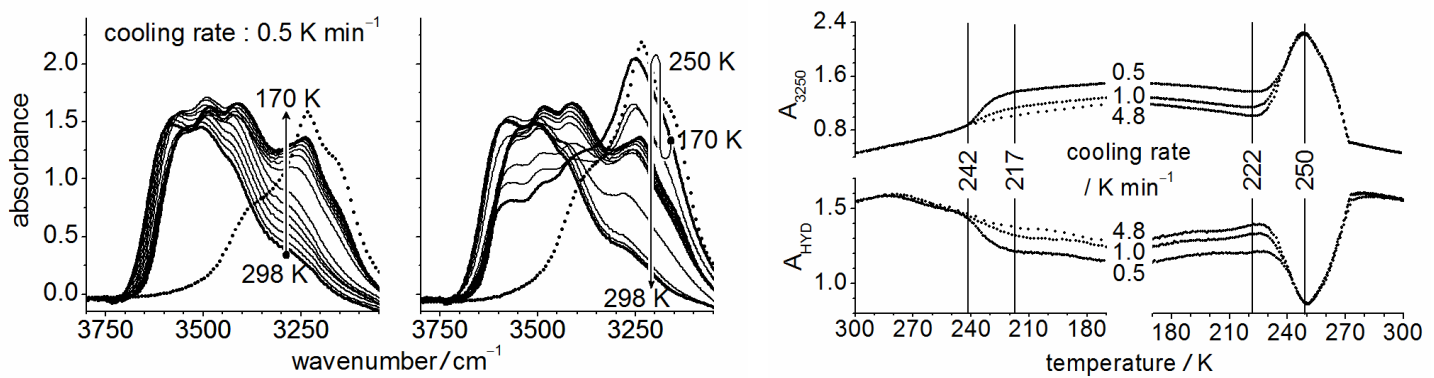

(C) PMA
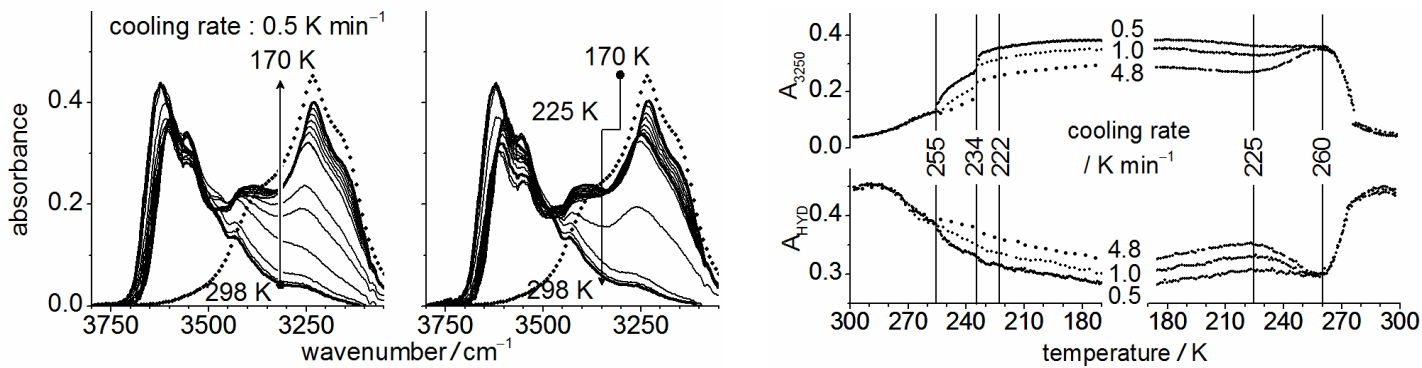

(D) PMMA
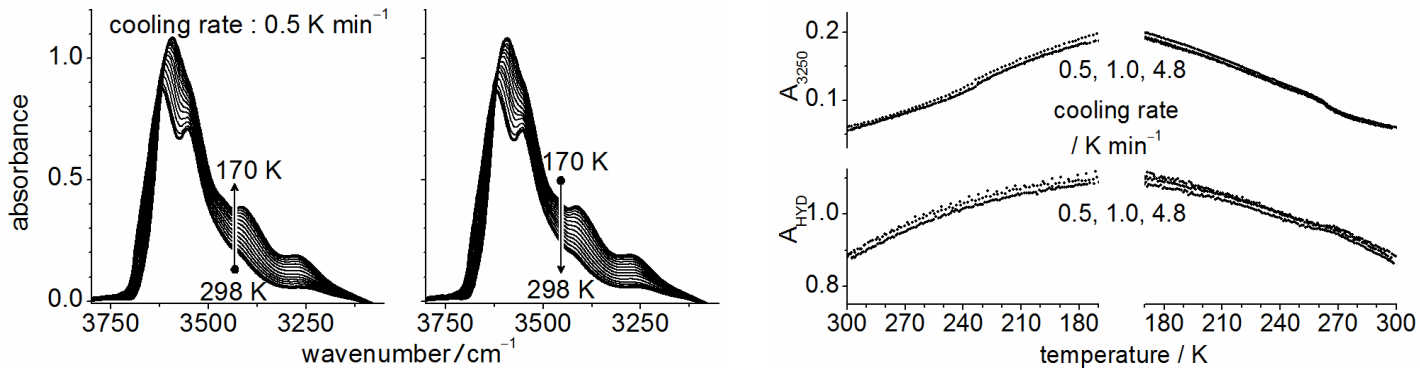

(E) Pure water
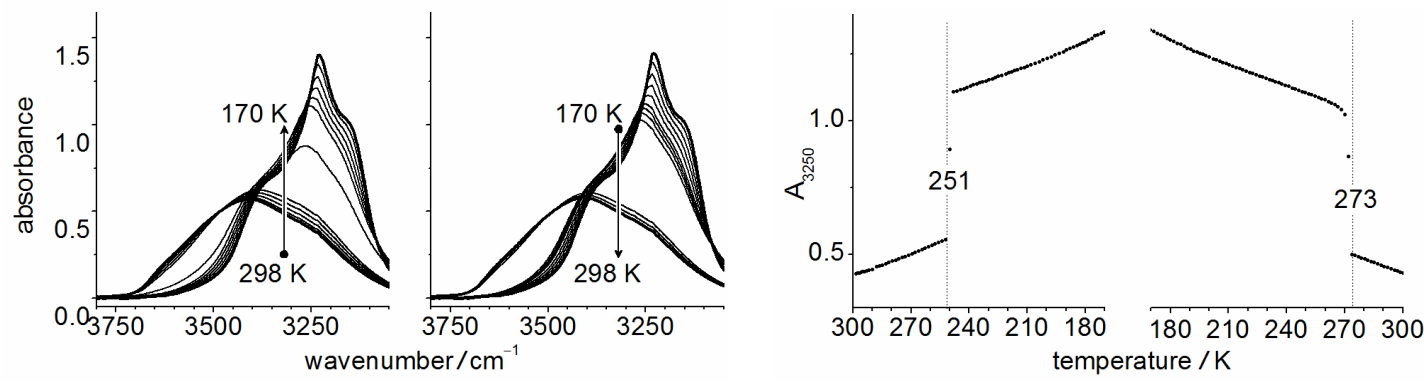

Figure 3. Left: Temperature-dependence of MIR spectra of water sorbed into the four polymers and pure water in the temperature range of $298-170 \mathrm{~K}$. Dotted line in panels $\mathrm{a}-\mathrm{C}$ is the spectrum of ice at $170 \mathrm{~K}$. Right: Temperature-dependences of $A_{3250}$ and $A_{H Y D}$ and that of $A_{3250}$ for pure water. (A) PMEA, (B) PTHFA, (C) PMA, (D) PMMA, and (E) pure water. 


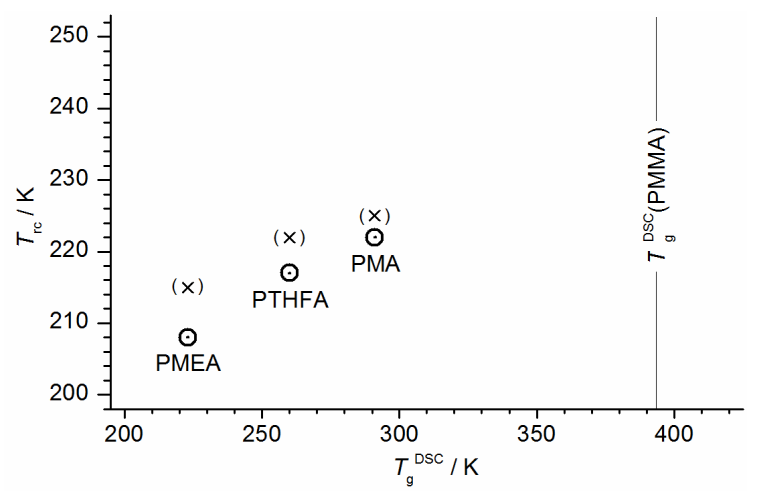

Figure 4. Relationship between $T_{\mathrm{g}}^{\mathrm{DSC}}$ of the polymer and $T_{\mathrm{rc}}$ of the sorbed water.

$\circ$ : recrystallization temperature, $T_{\mathrm{rc} .} \times$ : ending temperature of crystallization (crystal growth).

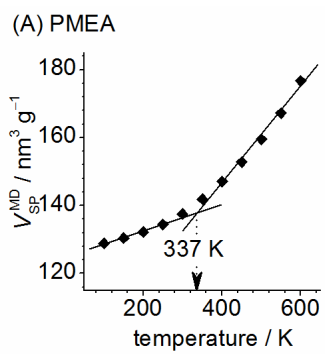

(B) PTHFA

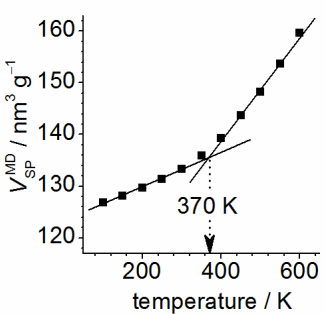

(C) PMA

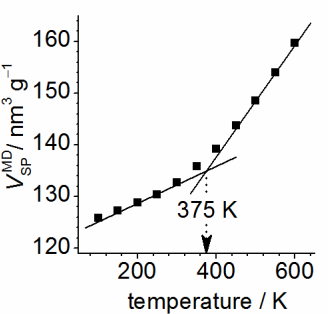

(D) PMMA

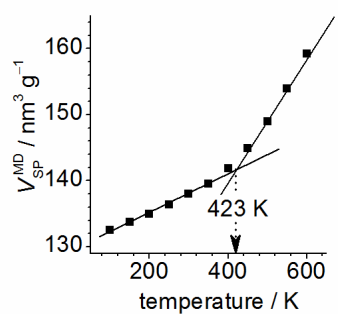

Figure 5. Temperature-dependence of specific volume $\left(V_{S P}^{\mathrm{MD}}\right)$ of the hydrated polymer. (A) PMEA, (B) PTHFA, (C) PMA, and (D) PMMA.
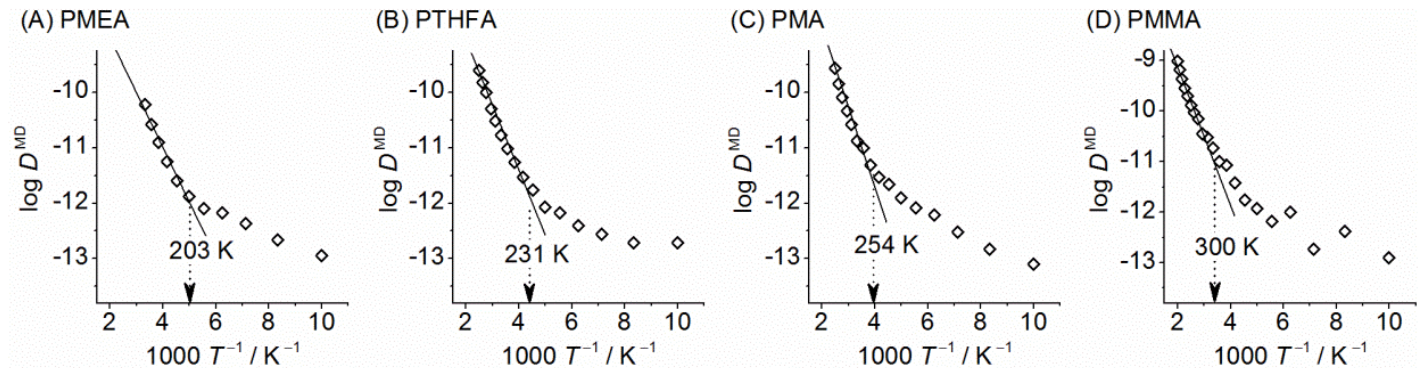

Figure 6. Arrhenius plot of the diffusion coefficient of the sorbed water. (A) PMEA, (B) PTHFA, (C) PMA, and (D) PMMA. 


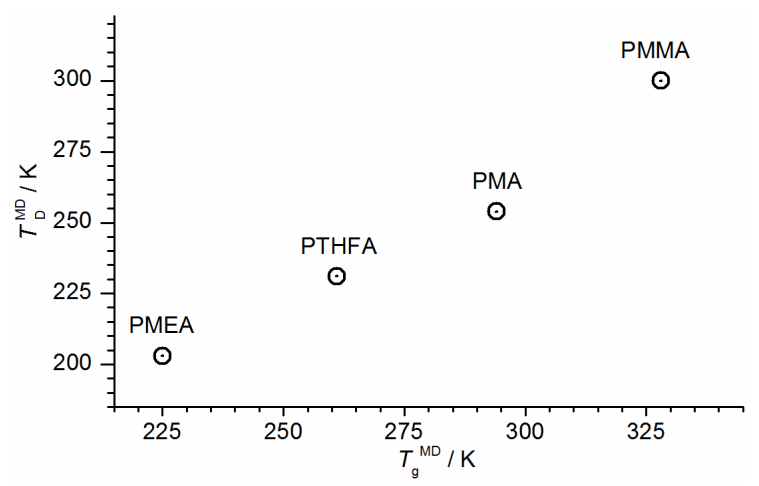

Figure 7. Relationship between $T_{\mathrm{g}}^{\mathrm{MD}}$ of the hydrated polymer and $T_{D}^{M D}$ of the sorbed water.
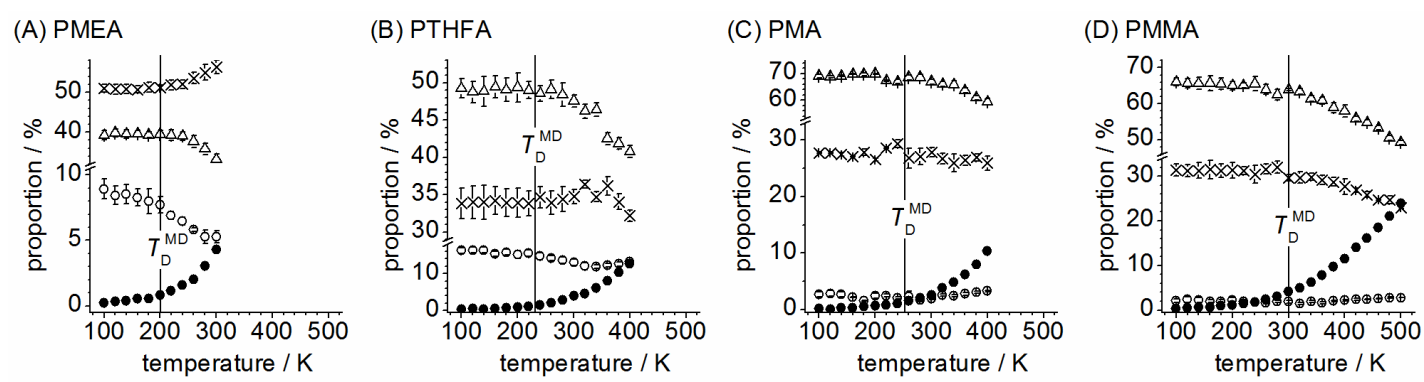

Figure 8. Temperature dependence of ratio of the hydrogen-bonding states $(x: \mathrm{O}-\mathrm{H}(1)$ in Figure $1, \Delta: \mathrm{O}-\mathrm{H}(2)$ in Figure 1, $\circ: \mathrm{O}-\mathrm{H}(3)$ in Figure 1, $\bullet: \mathrm{O}-\mathrm{H}(4)$ in Figure 1) for the sorbed water. (A) PMEA, (B) PTHFA, (C) PMA, and (D) PMMA. 